C

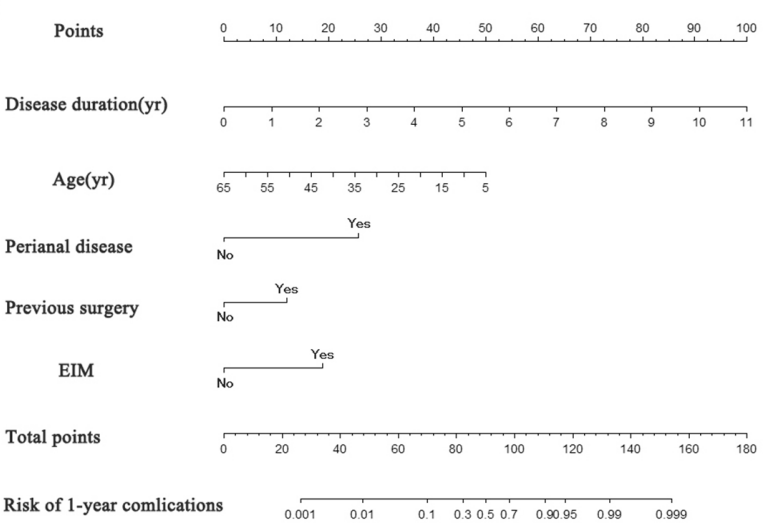

Abstract IDDF2020-ABS-0147 Figure 1C

Results The mean follow-up period was $53.45 \pm 12.81$ months. Of 614 eligible patients, $13.5 \%$ developed surgeryrelated complications, including stenosis, perforation, and severe gastrointestinal bleeding. We identified age (Odds ratio (OR) 0.914, $\mathrm{P}=0.004)$, disease duration (OR 2.675, $\mathrm{P}<0.001$ ), perianal disease ( $\mathrm{OR} 16.013, \mathrm{P}<0.001$ ), previous surgery (OR 3.652, $\mathrm{P}=0.003$ ), and extraintestinal manifestations $(\mathrm{OR} 7.625, \mathrm{P}=0.001)$ as significant independent factors associated with early-onset complications and developed a prognostic model ((figure 1A), A Prognostic model predicting complications leading to surgery within 1 year after diagnosis), whose predictive ability was appraised with AUC of 0.965 , specificity of $96.71 \%$, and sensitivity of $67.24 \%$. This model was validated with good discrimination (AUC of 0.933), and excellent calibration was demonstrated using the Hosmer-Lemeshow goodness-of-fit test ((figure 1B), HosmerLemeshow goodness-of-fit test demonstrating a good fit of this model). A nomogram was created to facilitate clinical bedside practice ((figure 1C) A nomogram predicting complications leading to surgery within 1 year after diagnosis in Crohn's disease patients).

Conclusions This validated prognostic model can effectively predict early-onset complications leading to surgery and screen aggressive $\mathrm{CD}$, enabling physicians to customize therapeutic strategies and monitor the intensive disease.

\section{IDDF2020-ABS-0148 PROGNOSTIC SIGNIFICANCE OF SIGNET RING CELL IN GASTRIC CANCER: THE LOWER PROPORTION, THE POORER SURVIVAL}

Yang Li*, Yantao Tian. Dept. of Pancreatic and Gastric Surgery, National Cancer Center/ National Clinical Research Center for Cancer/Cancer Hospital, Chinese Academy of Medical Sciences and Peking Union Medical College, China

\subsection{6/gutjnl-2020-IDDF.94}

Background The incidence rate of gastric cancer (GC) has been changed with decreasing intestinal-type GC and increasing of diffuse-type GC, including signet-ring cell (SRC) type. The carcinoma with SRC component more than $50 \%$ is defined as SRC carcinoma according to the pathological definition of WHO in 2010. However, the definition of gastric SRC is still controversial in past decades. And the prognosis affected by the proportion of SRC in GC is uncertain. This study compared the clinicopathological features and prognosis of gastric SRC with the various proportions of SRC.

Methods 1,069 patients who underwent gastrectomy for gastric signet ring cell cancer from 2011 to 2018 at the Chinese

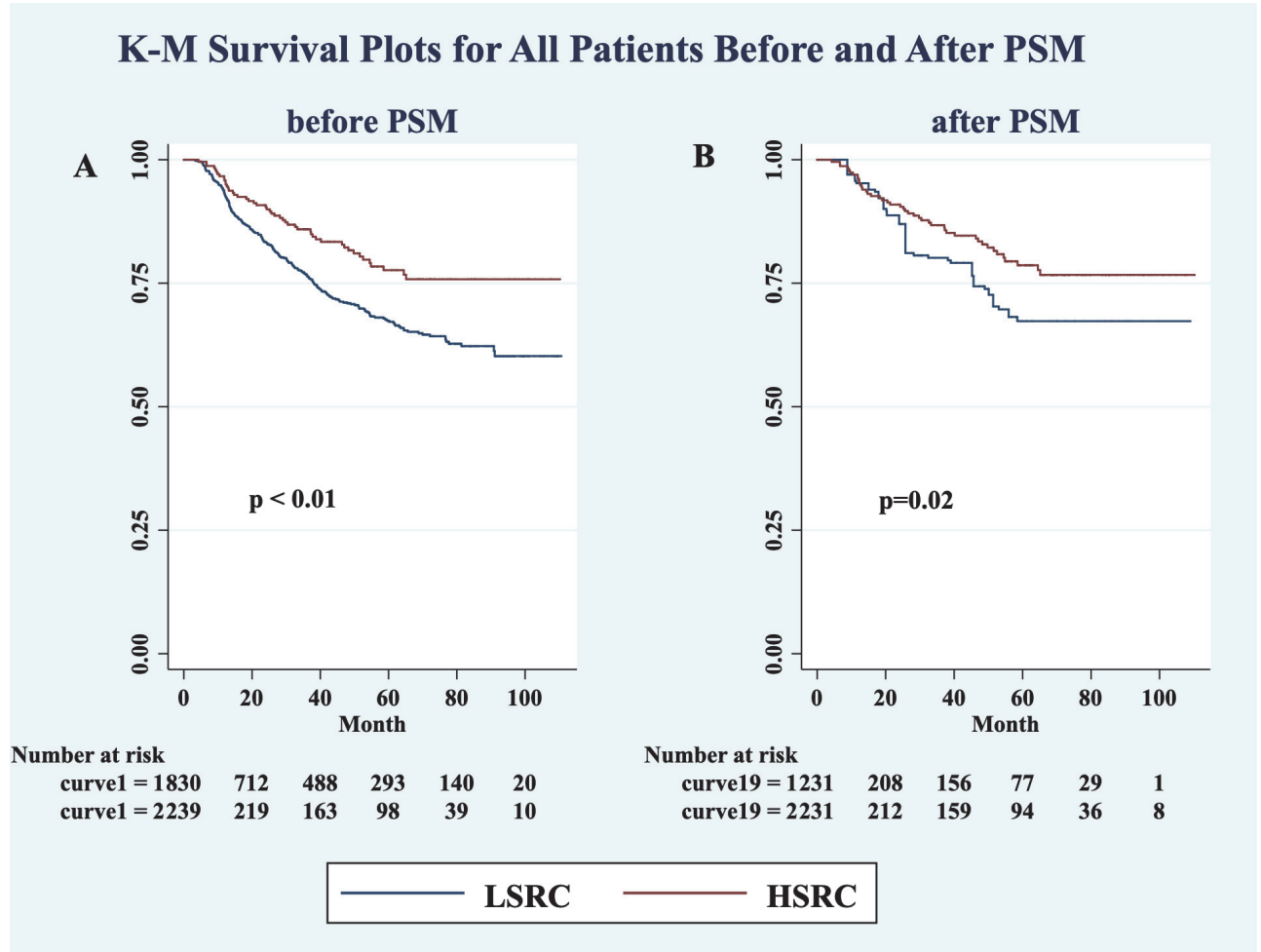

Abstract IDDF2020-ABS-0148 Figure 1 Survival plot for all patients before and after PSM 
Academy of Medical Science/National Cancer Center were compared for patient demographics, pathological characteristics, and treatments. Patients were divided into 2 groups as high proportion of SRC (HSRC) and low proportion of SRC (LSRC). Propensity Score Matching (PSM) was used to balance the sample size and bias.

Results Of 1,069 patients, 830 and 239 patients were divided into LSRC and HSRC groups separately. After matching, 462 patients (231 per group) were included in the analysis. 5-year OS was observed for LSRC and HSRC groups before matching $(67.4 \%$ vs $77.6 \%, \mathrm{P}<0.01)$, and after matching $(67.3 \%$ vs $78.6 \%, \mathrm{p}<0.05)$. At the multivariate analysis, $>60$ years old and tumor size $>4 \mathrm{~cm}$ were recognized as risk factors, and non-neoadjuvant chemotherapy was recognized as a protective factor. The HSRC was found as a protective factor after matching $(\mathrm{HR}=0.56,95 \% \mathrm{CI}: 0.38,0.84)$ for OS. The survival was better in the HSRC group than in the LSRC group. (figure 1)

Conclusions Our results confirm the prognostic differences between LSRC and HSRC groups, which means the proportion of SRC could affect the survival benefits of patients. Further clinical studies should be developed in the future to provide an appropriate treatment strategy for gastric SRC.

\section{IDDF2020-ABS-0149 LYMPHADENECTOMY WITH OPTIMAL REMOVED LYMPH NODES ASSOCIATED WITH IMPROVED STAGING AND SURVIVAL IN STAGE I-III GASTRIC CANCER: IS THERE AN OPTIMAL CUT-OFF POINT?}

Yang Li*. Dept. of Pancreatic and Gastric Surgery, National Cancer Center/National Clinical Research Center for Cancer/Cancer Hospital, Chinese Academy of Medical Sciences and Peking Union Medical College, China

10.1136/gutjnl-2020-IDDF.95
Background Gastric cancer is a common malignant tumor worldwide with lymph node metastases for which lymphadenectomy results in a survival benefit. Although lymphadenectomy with at least 16 removed lymph nodes (RLN) has been recommended in the procedure of gastrectomy by NCCN guideline to optimize node staging, the impact of the number of RLN on survival is uncertain.

Methods In this study, we sought to investigate the impact of the optimal number of RLN removed stratified by different stages on the prognosis of gastric cancer. 4,374 patients who underwent gastrectomy for gastric cancer from 2006 to 2016 at the Surveillance, Epidemiology and End Results Program (SEER) database were analyzed. Patients with M1 disease were excluded. The optimal threshold for the number of RLN was determined by the X-tile software. A competing risk analysis was performed to evaluate prognostic factors and to estimate subdistribution hazard ratios of mortality from gastric cancer. Standard survival methods and restricted cubic spline multivariable Cox regression models were applied.

Results Of 4,374 patients, mean age was 65.4 (13.6) and $2,493(57.0 \%)$ patients were male. Patients with $\leq 16$ RLN was 2,176 (49.8\%). 2,618 (59.9\%) patients were White and $574(13.1 \%)$ were Black. The optimal threshold of RLN was determined as 22 for all patients, and 29, 15, 22, 20, 27, 28, 39 for stage IA-IIIC separately. Compared to AJCC 8th staging, the lower hazard ratio (HR) of mortality and better survival was observed in the new classification of stages by optimal cut-off RLNs. As RLN increasing, the HR of mortality was continuously declined. (Figure 1)

Conclusions The optimal number of RLN appears to have prognostic implications on survival and improve the staging system. According to the characteristics of different stages, the optimal cut-off points are supposed to be individualized. This study suggests that the optimal number of RLN is supposed to be adopted in the staging system in the future.
(A)

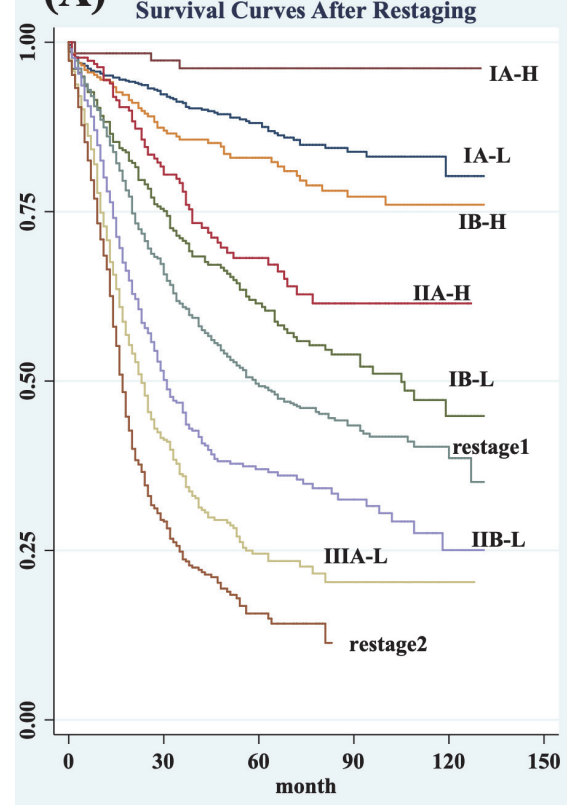

(B)

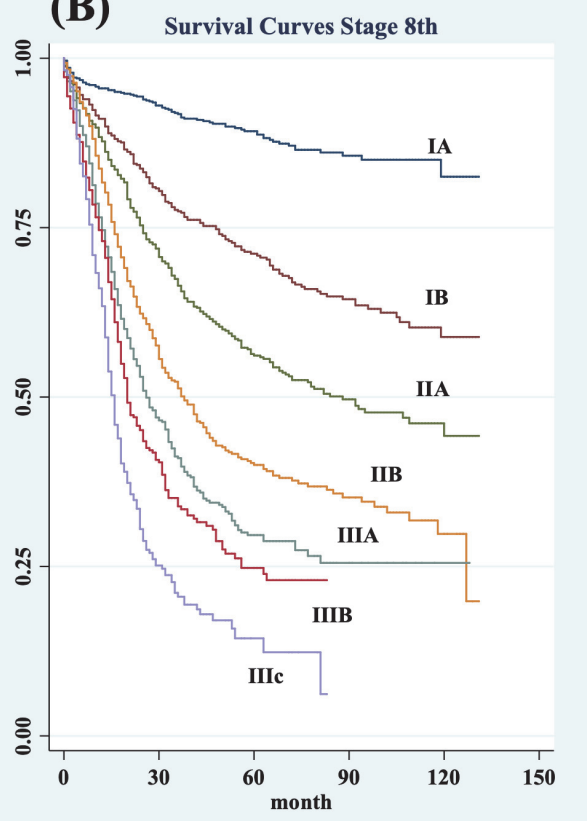

Abstract IDDF2020-ABS-0149 Figure 1 CCS curves by (A) restaging with stratification for number of optimal cut-off RLN and (B) AJCC 8th staging 